\title{
Raphaël Micheli, L'émotion argumentée. L'abolition de la peine de mort dans le débat parlementaire français
}

\section{Catherine Kerbrat-Orecchioni}

\author{
OpenEdition \\ Journals \\ Édition électronique \\ URL : http://journals.openedition.org/mots/20669 \\ DOI : 10.4000/mots.20669 \\ ISSN : 1960-6001 \\ Éditeur \\ ENS Éditions \\ Édition imprimée \\ Date de publication : 1 mai 2012 \\ Pagination : 137-141 \\ ISBN : 978-2-84788-356-5 \\ ISSN : 0243-6450
}

Référence électronique

Catherine Kerbrat-Orecchioni, « Raphaël Micheli, L'émotion argumentée. L'abolition de la peine de mort dans le débat parlementaire français », Mots. Les langages du politique [En ligne], $98 \mid 2012$, mis en ligne le 01 mai 2014, consulté le 19 avril 2019. URL : http://journals.openedition.org/mots/20669; DOI : $10.4000 /$ mots.20669 


\title{
Comptes rendus de lecture
}

\author{
L'émotion argumentée. L'abolition de la peine de mort \\ dans le débat parlementaire français \\ Raphaël Micheli \\ 2010, Paris, Cerf, 496 pages
}

Cet ouvrage de près de 500 pages se présente comme une défense et illustration de l'idée selon laquelle «les émotions contiennent une dimension intrinsèquement argumentable, qu'il est possible d'observer dans la matérialité du discours ». Plus généralement, « la capacité des émotions à générer des désaccords et leur propension à être argumentées par les locuteurs » constitueraient même « un aspect essentiel au fonctionnement des débats de société ». Cette thèse est elle-même très solidement argumentée tout au long de l'ouvrage, à partir de l'analyse minutieuse des principaux débats parlementaires sur la peine de mort qui ont marqué l'histoire de la France contemporaine (1791, $1848,1908,1981$ ) - débats qui comportent de par leur thème une composante émotionnelle particulièrement forte. Il s'agit donc pour l'auteur de suivre au fil de ces deux siècles la façon dont les orateurs s'y prennent pour construire des émotions telles que peur, pitié, indignation, honte, et quels arguments sont avancés en leur faveur ou défaveur. Les émotions sont donc envisagées comme étant non seulement mises au service de l'argumentation (approche «standard » du problème : l'appel à l'émotion comme instrument de persuasion), mais comme étant elles-mêmes objets d'une argumentation, de nature évidemment différente selon qu'elle s'inscrit dans le cadre d'une rhétorique abolitionniste ou anti-abolitionniste.

La question des émotions bénéficie incontestablement d'un certain « effet de mode» depuis une quinzaine d'années. Elle est ici abordée sous un angle neuf, qui permet d'enrichir considérablement la façon dont peut être conceptualisée l'articulation entre argumentation et émotion. Pour ce faire, l'investigation mobilise trois traditions disciplinaires : la rhétorique antique, les théories modernes de l'argumentation et les sciences du langage - avec quelques percées du côté de la sociologie (Boudon, Boltanski...) ou de la psychologie cognitive (Scherer, Ortony...). La diversité des références témoigne de l'étendue des lectures qui ont nourri ce travail, mais il s'agit toujours de «lectures ciblées » : l'auteur ne mobilise son érudition que dans la mesure où elle permet d'éclairer son questionnement théorique et son objectif descriptif.

L'étude s'organise en deux parties, la première servant à construire le cadre 
théorique et la deuxième procédant à la description du corpus. Elle est menée avec fermeté, l'exposé est toujours clair, la démonstration convaincante et l'écriture brillante ${ }^{1}$.

La première partie examine donc successivement les trois «traditions disciplinaires» annoncées, dont les «textes phares» sont passés au crible, afin de dégager en quoi ces réflexions antérieures peuvent venir alimenter la problématique présente :

- à propos de la tradition rhétorique, l'auteur insiste par exemple sur le fait qu'Aristote met déjà en évidence le rôle du jugement dans la formation des passions;

- à propos des théories modernes de l'argumentation, il passe en revue les différents courants : Toulmin, Perelman, la tradition normative et la théorie des fallacies, ainsi que les travaux de Christian Plantin qui ont ouvert la voie à cette exploration des relations entre émotion et argumentation;

- pour ce qui est de l'approche discursive et interactionniste des émotions, Raphaël Micheli envisage successivement le sort réservé par divers auteurs à l'émotion « éprouvée» (décrétée ici «non pertinente »), l'émotion « exprimée» (avec ses deux modalités du "dire» et du «montrer») et l'émotion "visée» (explicitement et implicitement), envisagée dans sa relation avec l'émotion « argumentée ». Il insiste sur la nécessité de prendre en compte, aux côtés des émotions " auto-attribuées», celles qui sont attribuées à autrui par l'orateur.

Ce parcours critique de la littérature débouche sur la proposition d'un «modèle d'analyse» permettant d'aborder le corpus, modèle centré sur la notion d'émotion attribuée, qui est retravaillée en relation avec la question de l'évaluation et de la légitimation de ces attributions. L'auteur introduit alors la notion fondamentale de «topos » et de « topique » : après une présentation très éclairante des différentes acceptions de ces notions, il formule l'idée que c'est essentiellement sur la base de «topiques» (façons particulières de conceptualiser et de présenter discursivement la situation évoquée) que sont légitimées les émotions associées, topiques dont il énumère, pour clore cette présentation du cadre théorique, les principaux paramètres.

La deuxième partie commence par un chapitre consacré au problème de la constitution du corpus, dont l'homogénéité repose, par-delà la dispersion temporelle, sur un principe de nature à la fois thématique et générique, puisqu'il s'agit de débats parlementaires autour de l'abolition de la peine de mort. Ce qui donne lieu à un développement sur les caractéristiques du genre discursif

1. La seule imperfection de l'ouvrage concerne les index : absence d'un index des notions, qui serait tout aussi utile que l'index des noms cités, lequel présente d'ailleurs certaines lacunes (par exemple : alors que C. Kerbrat-Orecchioni apparaît à dix reprises et pour quatre textes différents, son nom n'est mentionné dans l'index qu'à propos de la p.136, où la référence est d'ailleurs très incomplète - sans doute pour des raisons de réduction de la redondance : problème du système de références «à l'ancienne », sous la forme de notes en bas de page...) 
"débat parlementaire », développement qui pose le problème suivant : en réalité, le corpus n'est pas constitué de débats mais de transcriptions de débats, ce qui n'est pas tout à fait la même chose. Raphaël Micheli s'en justifie longuement (p. 207-213), au nom de la « relative autonomie de l'écrit», voire de la "supériorité du canal graphique». Il est vrai que l'on ne pourra jamais savoir ce qui s'est exactement passé dans l'enceinte du Parlement en 1791, 1848 et 1908. On aimerait tout de même en savoir plus sur la façon dont ont été réalisées ces transcriptions, leur destination exacte, leur degré de fidélité supposée (pour ce qui est du débat de 1981, une comparaison entre le débat oral et le texte écrit qui en est tiré serait à cet égard très instructive)... Il est en tout cas certain que les débats oraux et les transcriptions officielles de ces débats constituent deux objets, voire deux «genres» distincts, qui s'opposent à la fois par leur matérialité discursive (les affirmations concernant l'importance de l'actio dans les débats ou «la diversité des matériaux sémiotiques de l'interaction » semblent dans ces conditions un peu «décalées»), leur degré d'interactivité (il n'en reste plus grand chose dans ces transcriptions, malgré les quelques didascalies insérées dans les tirades, telles que murmures / murmures surplusieurs bancs, assentiment sur d'autres ou Très bien! À l'extrême gauche), et bien sûr leur dispositif communicatif et leur mode de diffusion - en particulier: quelles sont exactement les différentes strates de destinataires «visés » par ces discours (et qu'il faut atteindre émotionnellement) dans ces différents cas de figure (transcriptions écrites vs débats oraux, mais aussi débats antérieurs vs postérieurs à l'irruption des moyens audiovisuels)?

Cette réserve mise à part et le corpus étant ce qu'il est, on reste admiratif devant la richesse, la finesse et la pertinence des analyses présentées dans cette deuxième partie, où sont décortiqués successivement les quatre débats (chap. 6 à 9), et surmontées avec maestria les nombreuses difficultés que l'on rencontre lorsque l'on se trouve confronté à un matériel à analyser aussi considérable :

- problème de la perspective diachronique : il faut tenir compte du fait que chacun des quatre débats se déroule dans un contexte politique et idéologique particulier, et montrer que les topiques investies sont à la fois mouvantes et stables : le travail de Raphaël Micheli parvient à rendre compte avec une grande subtilité des ruptures aussi bien que des continuités dans les argumentaires développés durant ces deux siècles de débats (en débusquant les renvois intertextuels d'un débat à l'autre);

- chacun des débats offre une matière riche et complexe, impliquant différents orateurs : il faut nécessairement trier dans la masse, ramener la diversité des voix à un affrontement binaire entre le camp des abolitionnistes et celui des anti-abolitionnistes (notion d' "ethos de groupe ») tout en signalant les particularités de certaines voix individuelles (Robespierre, Badinter), choisir les extraits jugés particulièrement représentatifs des topiques et des argumentaires...; 
- il faut ensuite à la fois effectuer des analyses de détail (portant sur des faits aussi bien lexicaux que morphosyntaxiques ou rhétorico-stylistiques - voir par exemple les développements concernant les questions rhétoriques ou les figures d'analogie) et dégager des généralités sur la construction des émotions dans les différents sous-corpus. Par exemple:

a) dans le débat de 1791 : affrontements autour du problème de l'utilité sociale de la peine de mort, exploitation de la topique du spectacle de l'exécution du côté des abolitionnistes, appel à la peur du côté des antiabolitionnistes...;

b) dans le débat de 1848 : «question du droit » vs argument de la «sécurité de tous »; émergence de la figure du peuple, placée au cœur de la topique des deux camps mais avec des évaluations opposées...;

c) dans le débat de 1908 : montée en puissance de la figure de la «victime », construction argumentative de la pitié, requalification des coupables en êtres souffrants...;

d) dans le débat de 1981 : stratégie "pathos contre pathos », abolitionnistes et anti-abolitionnistes face à la honte et la culpabilité...

Le dernier chapitre de l'ouvrage récapitule de façon très claire les résultats obtenus tant au niveau théorique que méthodologique et descriptif. Le bilan est en effet extrêmement positif: d'un point de vue théorique, l'ouvrage renouvelle et enrichit de façon décisive la réflexion sur la façon dont s'articulent l'émotion et l'argumentation dans l'exercice de la parole et plus largement, le pathos, le logos et l'ethos; d'un point de vue méthodologique, l'étude est exemplaire par sa rigueur et son souci de toujours expliciter ses choix et procédures; d'un point de vue descriptif, elle scrute la matière de ces débats avec une attention et une intelligence du texte qui fait que cette succession d'analyses pourtant austères se lit presque comme un feuilleton.

La seule petite frustration que l'on éprouve tient comme on l'a dit plus haut au fait que ce travail se fait à partir non de la matière vive des débats (cela n'eût été possible que pour le dernier des quatre) mais de reproductions écrites, ce qui impose à l'analyse certaines limites : on n'a pas accès à la façon dont les émotions sont sémiotisées, mais seulement à la façon dont elles sont verbalisées, ce qui est certes l'essentiel dans la perspective d'une approche argumentative, mais aboutit à un certain nivellement des voix et des éthè des différents orateurs. On n'a pas accès non plus à la dimension proprement interactive de ces débats : leurs composantes dialogiques sont brillamment analysées (fonctionnements intertextuels, "forte polémicité » de ces débats - en général le discours anti-abolitionniste est présenté comme "répondant» globalement au discours abolitionniste mais l'ordre est parfois inversé, on aimerait savoir si cela correspond à quelque différence dans le «script » du débat et son organisation séquentielle); mais non leur fonctionnement dialogal sous la forme par exemple de négociations locales des émotions auto- et allo-attribuées (ce 
sont plus des types de discours que des locuteurs individuels qui sont présentés comme s'affrontant). Enfin, la nature du corpus et de l'orientation théorique entraînent une certaine banalisation de la strate émotionnelle du discours : tout en admettant l'idée que les contenus émotionnels sont en partie rationalisables et argumentables, on peut aussi estimer qu'ils présentent à tous les niveaux certaines spécificités qui sont quelque peu « gommées» dans l'étude de Micheli (entre autres : la question de l'«éprouvé» peut difficilement être évacuée comme non pertinente étant donné l'importance pour l'auditoire de l' " effet-de-sincérité » des émotions exprimées, comme l'ont montré par exemple certaines études de l'épisode de la «saine colère» dans le débat Royal-Sarkozy de 2007).

L'étude s'achève sur l'idée qu'à partir de la fin du $20^{e}$ siècle, la question des victimes est devenue centrale. Sans doute. Mais cette évolution des mentalités n'aurait-elle pas dû profiter justement au camp des anti-abolitionnistes? Le fait que les abolitionnistes l'aient emporté en 1981 tient à de nombreux facteurs, dont le contexte politique (on sait que le vote de l'Assemblée se fit à l'encontre de l'opinion majoritaire des Français), mais aussi sans doute la puissance de l'éloquence de Robert Badinter, qui fut au cœur de ce débat non seulement du fait de son statut de ministre de la Justice et de garde des Sceaux mais aussi par son talent dans le maniement de l'argumentation et de la rhétorique émotionnelle, par sa force de conviction liée à celle de son engagement, et qui restera dans les mémoires et les annales comme celui qui a assuré la victoire du camp abolitionniste.

En tout état de cause, ce n'est pas avec les seules armes de la linguistique que l'on peut expliquer de tels événements socio-historiques. On dira simplement que ces armes, Raphaël Micheli les manie avec rigueur et talent, dans cet ouvrage impressionnant par sa richesse et sa cohérence; ouvrage de bout en bout passionnant, qui constitue une contribution décisive à l'analyse du discours et plus particulièrement à l'étude des «logiques affectives».

Catherine Kerbrat-Orecchioni

Université Lyon 2, ICAR

catherine.kerbrat-orecchioni@univ-lyon2.fr 